\title{
A CRITICAL SET WITH NONNULL IMAGE HAS LARGE HAUSDORFF DIMENSION
}

\author{
ALEC NORTON
}

\begin{abstract}
The question of how complicated a critical set must be to have a nonnull image is answered by relating its Hausdorff dimension to the (Hölder) differentiability of the map. This leads to a new extension of the Morse-Sard Theorem. The main tool is an extended version of Morse's Lemma.
\end{abstract}

1. Introduction. In $1935 \mathrm{H}$. Whitney [W] published his celebrated example of a $C^{1}$ function $f: \mathbf{R}^{2} \rightarrow \mathbf{R}$ not constant on a connected critical set $A$. The conditions allowing such an example to occur (or, more generally, the conditions under which images of critical sets can have positive measure) were at that time poorly understood.

The partial results then known were noted by Whitney in the same paper: such an example cannot occur if either

(1) every pair of points in $A$ is connected by a rectifiable arc lying in $A$ (a result of W. M. Whyburn), or

(2) $f$ is sufficiently differentiable (a result of M. Morse and A. Sard).

During the ensuing decade a more complete picture was provided by results in the direction of (2), first with A. P. Morse's work [M] improving "sufficiently differentiable" in this case to $C^{2}$, and then by A. Sard [S1], who extended this result to the vector-valued case with the Morse-Sard Theorem. Since then, Sard [S4], Federer $[\mathbf{F}]$, and lately Yomdin $[\mathbf{Y}]$ have further generalized the now classical Morse-Sard Theorem to obtain a sharp estimate of the size (suitably measured by Hausdorff or entropy dimension) of the image of a set of rank $r$ points in terms of the (Hölder) differentiability class of $f$ and the dimension of the domain.

Relatively less has been done to follow up (1) of Whyburn. The question Whyburn's work posed was in fact asked by Whitney in the same paper [W]:

Given a function $f$, how far from rectifiable must a closed connected set be to be a critical set for $f$ on which $f$ is not constant?

The purpose of this paper is to give a satisfactory answer to this question (Corollaries 2 and 3), by using Hausdorff dimension to measure the "distance" of a set from rectifiability. Namely, (a) if $f: \mathbf{R}^{m} \rightarrow \mathbf{R}$ is of class $C^{k+\beta}$, then such a set must have Hausdorff dimension at least $k+\beta$, and (b) such a set must have a pair of points which do not both lie in any connected subset with Hausdorff dimension less than $k+\beta$. In the course of finding this answer we also provide a further generalization of the Morse-Sard Theorem (Theorem 1), and extract Whyburn's result (1) as a corollary.

Received by the editors March 7, 1985 and, in revised form, August 15, 1985.

1980 Mathematics Subject Classification. Primary 26B35; Secondary 58C25, 28A25, 49F20. 
A partial answer to Whitney's question was given in 1958 by Sard [S3] who provided effectively the answer (a) above for the case of integer differentiability classes $C^{k}$. This paper provides an extension of Sard's results to more arbitrary differentiability classes and thus illuminates more clearly the sensitive relation between the (real-valued) Hausdorff dimension of a critical set and the (real-valued) differentiability class of the corresponding function.

We first found these results for real-valued functions by using entropy dimension to analyze critical sets. The improvement to Hausdorff dimension was inspired by [S3], which also provided Federer's key ingredient in the proof of the vector-valued case. Our main improvement is the proof of the key Morse Lemma, on which Sard and Federer both rely, in the case of more general differentiability classes.

In $\S 2$ we give the necessary definitions, state the theorems in $\S 3$, prove a generalized version of Morse's Lemma in $\S 4$, and provide the proofs of the main theorem, Theorem 1, in $\S 5$. $\S 6$ gives some examples showing that the hypotheses of Theorems 1 and 2 cannot be weakened.

ACKNOWLEDGEMENTS. I thank J. Harrison for providing, with her work on Denjoy fractals $[\mathbf{H}]$, the inspiration that Hölder differentiability and Hausdorff dimension are related. Thanks are also due to C. Pugh for his lectures on the Morse Lemma and for useful comments.

2. Definitions. Given a metric space $X$, for each positive real number $s$ there is a corresponding $s$-dimensional Hausdorff measure $\not^{s}$ defined as follows. Let $B \subset X$ be an arbitrary set. For $\alpha>0$, let

$$
\mathfrak{H}_{\alpha}^{s}(B)=\inf \sum_{i}\left[\operatorname{diam}\left(B_{i}\right)\right]^{s},
$$

where the infimum is taken over all countable decompositions $\left\{B_{i}\right\}$ of $B$ such that $\operatorname{diam}\left(B_{i}\right)<\alpha$ for each $i$. $\left(\left\{B_{i}\right\}\right.$ is a decomposition of $B$ if $\bigcup B_{i}=B$.) Then $\nvdash^{s}(B)$ is defined to be the (possibly infinite) limit of $\varkappa_{\alpha}^{s}(B)$ as $\alpha$ tends to zero from above. (The limit exists because $\mathfrak{H}_{\alpha}^{s}(B)$ is nondecreasing as $\alpha$ decreases.)

We say (after Sard [S3]) that $B$ is $s$-null if $\not^{s}(B)=0, s$-finite if $\mathfrak{H}^{s}(B)<\infty$, and $s$-sigmafinite if $B$ is a countable union of $s$-finite sets.

In $\mathbf{R}^{n}$, when there is no danger of confusion, null will mean $n$-null, and this coincides with $n$-dimensional Lebesgue measure zero [S2].

A set $B$ has Hausdorff dimension $s$, denoted $\operatorname{HD}(B)=s$, iff $\mathcal{K}^{r}(B)=0$ for all $r>s$ and $\mathcal{H}^{r}(B)=\infty$ for all $r<s$. $\left(\mathscr{H}^{s}(B)\right.$ itself may be 0 , finite, or infinite.) Note [HW]: $\mathscr{H}^{r}(B)<\infty$ implies $\mathcal{H}^{s}(B)=0$ for $s>r$, and $\nvdash^{r}(B)>0$ implies $\nvdash^{s}(B)=\infty$ for $s<r$.

For $m, n, k \in \mathbf{Z}^{+}$, a function $f: \mathbf{R}^{m} \rightarrow \mathbf{R}^{n}$ is of class $C^{k}\left(f \in C^{k}\left(\mathbf{R}^{m}, \mathbf{R}^{n}\right)\right)$ if $f$ together with all its partial derivatives of order $\leq k$ are defined and continuous on $\mathbf{R}^{m}$. For $0 \leq \beta<1, f$ is of class $C^{k+\beta}$ if $f$ is $C^{k}$ and the $k$ th derivative $D^{k} f$ satisfies a $\beta$-Hölder condition: for every compact neighborhood $U$ there is an $M>0$ such that

$$
\left|D^{k} f(x)-D^{k} f(y)\right| \leq M|x-y|^{\beta} \quad \text { for all } x, y \in U .
$$

$f$ is of class $C^{k+\text { Lip }}$ if $f \in C^{k}$ and $f$ satisfies (1) with $\beta=1$.

$f$ is of class $C^{k+\beta+}$ if $f \in C^{k}$ and $D^{k} f$ satisfies a strong $\beta$-Hölder condition: for every compact neighborhood $U$ there is a continuous nonnegative monotone 
function $\varepsilon: \mathbf{R} \rightarrow \mathbf{R}$ with $\varepsilon(0)=0$ and

$$
\left|D^{k} f(x)-D^{k} f(y)\right| \leq \varepsilon(|x-y|)|x-y|^{\beta} \quad \text { for all } x, y \in U .
$$

Note now that $C^{k+0+}=C^{k}=C^{k+0}$, and $\bigcup_{\beta^{\prime}>\beta} C^{k+\beta^{\prime}} \varsubsetneqq C^{k+\beta+} \varsubsetneqq C^{k+\beta}$.

$A$ is a set of rank $r$ for $f$ if the rank of $D f_{x}$ is at most $r$ for every $x \in A$. $A$ is a critical set for $f$ if it is a set of rank $n-1$.

3. Theorems. In all that follows, $m, n$ and $k$ denote positive integers, $r$ is a nonnegative integer, and $\beta \in[0,1)$.

THEOREM 1. Let $f: \mathbf{R}^{m} \rightarrow \mathbf{R}^{n}$ and $A$ be a set of rank $r$ for $f$, with $r<n$.

(i) If $f \in C^{k+\beta+}$ and $A$ is $((k+\beta)(n-r)+r)$-sigmafinite, then $f(A)$ is null (= $n$-null).

(ii) If $f \in C^{k+\beta}$ and $A$ is $((k+\beta)(n-r)+r)$-null, then $f(A)$ is null.

(iii) If $f \in C^{k+\mathrm{Lip}}$ and $A$ is $((k+1)(n-r)+r)$-null, then $f(A)$ is null.

Setting $r=n-1$ yields our central

THEOREM 2. Let $f: \mathbf{R}^{m} \rightarrow \mathbf{R}^{n}$ and $A$ be a critical set for $f$.

(i) If $f \in C^{k+\beta+}$ and $A$ is $(k+\beta+n-1)$-sigmafinite, then $f(A)$ is null.

(ii) If $f \in C^{k+\beta}$ and $A$ is $(k+\beta+n-1)$-null, then $f(A)$ is null.

(iii) If $f \in C^{k+\operatorname{Lip}}$ and $A$ is $(k+n)$-null, then $f(A)$ is null.

REMARKS. (a) Theorem 1 is dual to Federer's theorem [F, §3.4.4] (also [Y]): If $f \in C^{k}\left(\mathbf{R}^{m}, Y\right)$ ( $Y$ any normed vector space), and $A$ has rank $r$ for $f$, then $f(A)$ is $(r+(m-r) / k)$-null. Instead of asking how small the image is of an arbitrary ( $m$-sigmafinite) rank $r$ set, we are asking how small the rank $r$ set must be to have an image of fixed size ( $n$-null). Accordingly, $m$ does not appear in the statement of Theorem 1, while $n$ does not appear in Federer's statement. However, we are not dually free to replace the domain $\mathbf{R}^{m}$ in Theorem 1 by an arbitrary Banach space $Y$ since our proof depends strongly on the finite dimensionality of the domain.

A generalization of both theorems, with the aim of eliminating mention of both $m$ and $n$ might be: If $A$ has rank $r$ for $f \in C^{k+\beta}$ and is $s$-null, then $f(A)$ is $(r+(s-r) /(k+\beta))$-null. However, the proof of such a theorem using our methods seems to be blocked by the absence of a Fubini theorem for arbitrary Hausdorff measures. (Federer has given a Fubini counterexample, quoted in [S4].)

(b) Theorem 1(iii) is a strengthening of (ii) in the case $\beta=0$ and $k \geq 2$; it follows (see below) from the proof of (ii) with $\beta=1$. The presence of this case here and in the sequel is required by the ambiguity in the meaning of $C^{k+\beta}$ when $\beta=1$. The notation $C^{k, \beta}$ would have removed this ambiguity by distinguishing $C^{k+1,0}$ from $C^{k, 1}$, but the additive notation is firmly in place in the literature and partly justified by these theorems themselves (and ultimately by Taylor's Theorem).

Corollary 1 (MORSE-SARD Theorem). If $f \in C^{k}\left(\mathbf{R}^{m}, \mathbf{R}^{n}\right), A$ is a critical set for $f$ and $k=\max \{1, m-n+1\}$, then $f(A)$ is null $(=n$-null $)$.

Proof. Set $\beta=0$ in Theorem 2(i) and note that any subset of $\mathbf{R}^{m}$ is $m$ sigmafinite. 
From the definition of Hausdorff dimension and Theorem 2(ii) with $n=1$, we obtain

COROLlaRY 2. If $f \in C^{k+\beta}\left(\mathbf{R}^{n}, \mathbf{R}\right), A$ is critical for $f$, and $\operatorname{HD}(A)<k+\beta$, then $f(A)$ is null.

Hence if $A$ is connected, then $f$ is constant on $A$.

Proof. If $A$ is connected, so is $f(A)$. Hence $f(A)$ is an interval. But a (nonempty) null interval is a point.

An even better answer to Whitney's question is now easily obtained:

COROLlaRY 3. If $f \in C^{k+\beta}\left(\mathbf{R}^{m}, \mathbf{R}\right), A$ is a connected critical set for $f$, and if for every $x, y \in A$ there is a connected set $S \subset A$ with $x, y \in S$ and $\operatorname{HD}(S)<k+\beta$, then $f$ is constant on $A$.

Proof. For $x, y \in A$, choose such a set $S$. By Corollary $2, f$ is constant on $S$. Hence $f(x)=f(y)$.

Almost a special case of Corollary 3 is

COROllary 4 (WhyBurN). If $f: \mathbf{R}^{m} \rightarrow \mathbf{R}$ is $C^{1}$ and $A$ is a critical set for $f$ which is arcwise connected by rectifiable arcs, then $f$ is constant on $A$.

PROOF. If $\gamma$ is a rectifiable arc, it is easy to check that it is 1-finite. If it connects $x$ to $y$ in $A$, then by Theorem 2(i), $f(\gamma)$ is null. Hence $f$ is constant on $\gamma$ (as in the proof of Corollary 2), so $f(x)=f(y)$.

We remark here that such a set $A$ might itself have quite large Hausdorff dimension. For example, $A$ might be an open ball, or a cone in $\mathbf{R}^{m}$ over a large set in $\mathbf{R}^{m-1} \times 0$. The important requirement for Corollaries 3 and 4 is that pairs of points in $A$ be connected by subsets of small dimension.

\section{Generalized Morse Theorem.}

THEOREM 3. (For the ordinary Morse theorem see $[\mathbf{M}]$ or $[\mathbf{S t}]$.) Let $m, k$ be positive integers and $\beta \in[0,1)$. Let $A$ be a subset of $\mathbf{R}^{m}$.

(i) There are subsets $A_{i}, i=0,1,2, \ldots$, of $A$ with $A=\bigcup A_{i}$ such that $A_{0}$ is countable and any $f \in C^{k+\beta+}\left(\mathbf{R}^{m}, \mathbf{R}\right)$ critical on $A$ satisfies, for each $i$ :

$$
|f(x)-f(y)| \leq \varepsilon_{i}(|x-y|)|x-y|^{k+\beta} \quad \text { for all } x, y \in A_{i},
$$

where $\varepsilon_{i} \geq 0$ is some monotone continuous function $\mathbf{R} \rightarrow \mathbf{R}$ such that $\varepsilon_{i}(0)=0$.

(ii) There are subsets $A_{i}^{\prime}, i=0,1,2, \ldots$, of $A$ with $A=\bigcup A_{i}^{\prime}$ such that $A_{0}^{\prime}$ is countable and any $f \in C^{k+\beta}\left(\mathbf{R}^{m}, \mathbf{R}\right)$ critical on $A$ satisfies, for each $i$,

$$
|f(x)-f(y)| \leq M_{i}|x-y|^{k+\beta} \quad \text { for all } x, y \in A_{i}^{\prime} \text {, and some } M_{i}>0 \text {. }
$$

(iii) There are subsets $A_{i}^{\prime \prime}, i=0,1,2, \ldots$, of $A$ with $A=\bigcup A_{i}^{\prime \prime}$ such that $A_{0}^{\prime \prime}$ is countable and any $f \in C^{k+\operatorname{Lip}}\left(\mathbf{R}^{m}, \mathbf{R}\right)$ critical on $A$ satisfies, for each $i$,

$$
|f(x)-f(y)| \leq M_{i}|x-y|^{k+1} \quad \text { for all } x, y \in A_{i}^{\prime \prime} \text {, and some } M_{i}>0 \text {. }
$$

Such a decomposition of $A$ is called a Morse decomposition, and depends on $m$, $k$, and $\beta$, but not on the function $f$.

PROOF. We prove (i); the proofs of (ii) and (iii) are similar. 
The proof depends on

THE GENERALIZED MORSE VANISHING LEMMA. Let $m$ be a positive integer, $k$ a nonnegative integer, and $\beta \in[0,1)$. Then any set $A$ in $\mathbf{R}^{m}$ can be decomposed into a countable union $A=\bigcup A_{i}(i=0,1,2, \ldots)$ where $A_{0}$ is countable, and $A_{i} \subset B_{i}$, a $C^{1}$-embedded compact disk of dimension $\leq m$, such that every $f \in C^{k+\beta+}\left(\mathbf{R}^{m}, \mathbf{R}\right)$ vanishing on $A$ satisfies, for each $i$,

$$
|f(x)-f(y)| \leq \varepsilon_{i}(|x-y|)|x-y|^{k+\beta} \quad \text { for all } y \in A_{i} \text { and } x \in B_{i},
$$

where $\varepsilon_{i} \geq 0$ is some monotone continuous function $\mathbf{R} \rightarrow \mathbf{R}$ such that $\varepsilon_{i}(0)=0$.

Proof. Fix $\beta$. The proof is by induction on $m$ and $k$. Let $\langle m, k\rangle$ stand for the statement of the theorem for $\mathbf{R}^{m}$ and $C^{k+\beta+}$. We will prove $\langle n, 0\rangle$ for all $n$, $\langle 1, k\rangle$ for all $k$, and $(\langle n-1, k\rangle \&\langle n, k-1\rangle \Rightarrow\langle n, k\rangle)$ to establish the theorem for all $n \geq 1, k \geq 0$.

(a) Proof of $\langle n, 0\rangle$ for all $n$. If $f \in C^{\beta+}\left(\mathbf{R}^{n}, \mathbf{R}\right)$, then by definition there is a monotone nonnegative continuous function $\delta_{i}: \mathbf{R} \rightarrow \mathbf{R}$ such that $\delta_{i}(0)=0$ and $|f(x)-f(y)| \leq \delta_{i}(|x-y|)|x-y|^{\beta}$ on the closed ball $B(0, i)$ of radius $i$ centered at the origin. Now let $A_{0}=\varnothing$ and $A_{i}=A \cap B(0, i)$ for each $i$. Then $\varepsilon_{i}=\delta_{i}$ are the required functions.

(b) Proof of $\langle 1, k\rangle$ for any $k$. Let $A^{*}$ be the set of condensation points of $A$ ( $x \in A$ is a condensation point if every neighborhood of $x$ meets $A$ in uncountably many points). Let $A_{0}=A \backslash A^{*}$ (a countable set), and $A_{i}=A^{*} \cap(-i, i)$. From Taylor's theorem,

$$
\begin{aligned}
f(x)=f(y)+f^{\prime}(y)(x-y) & +\cdots+f^{(k-1)}(y)(x-y)^{k-1} /(k-1) ! \\
& +(x-y)^{k} f^{(k)}(y+\theta(x-y)) / k ! \quad \text { for some } \theta \in[0,1]
\end{aligned}
$$

when $x$ is near $y \in A_{i}$. Since $f=0$ on $A_{i}$ and $A_{i}$ has no isolated points, $f^{\prime}=0$ on $A_{i}$. Similarly, $f^{\prime \prime}=f^{(3)}=\cdots=f^{(k)}=0$ on $A_{i}$. Hence

$$
f(x)=f(y)+(x-y)^{k} f^{(k)}(y+\theta(x-y)) / k ! .
$$

It will suffice to show that $|f(x)-f(y)| /|x-y|^{k+\beta}$ tends to zero as $|x-y|$ tends to zero for $x \in B_{i}=[-i, i]$ and $y \in A_{i}$. Now

$$
\begin{aligned}
\frac{|f(x)-f(y)|}{|x-y|^{k+\beta}} & =\frac{\left|f^{(k)}(y+\theta(x-y))\right|}{k !|x-y|^{\beta}}=\frac{\left|f^{(k)}(y+\theta(x-y))-f^{(k)}(y)\right|}{k !|x-y|^{\beta}} \text { if } y \in A_{i}, \\
& =\frac{\theta^{\beta}}{k !} \frac{\left|f^{(k)}(y+\theta(x-y))-f^{(k)}(y)\right|}{|(y+\theta(x-y))-y|^{\beta}} \rightarrow 0
\end{aligned}
$$

as $|x-y| \rightarrow 0$, from the definition of $C^{k+\beta+}$.

(c) Induction Step. Suppose $\langle n, k-1\rangle$ and $\langle n-1, k\rangle$ are true for $k \geq 1, n \geq 2$.

Let $f \in C^{k+\beta+}\left(\mathbf{R}^{n}, \mathbf{R}\right)$. Let

$U=\left\{x \in A\right.$ : every $g \in C^{k+\beta+}\left(\mathbf{R}^{n}, \mathbf{R}\right)$ vanishing on $A$ is critical at $\left.x\right\}$ and

$V=A \backslash U$.

We prove the result separately for $U$ and $V$.

On $U$ : Since $f$ vanishes on $A, f$ is critical on $U$, so for each $j=1, \ldots, n$, $D_{j} f=\partial f / \partial x_{j}$ vanishes on $U$, and these functions are all of class $C^{k-1+\beta+}$. Hence 
by the $\langle n, k-1\rangle$ hypothesis there is a Morse decomposition $U=\bigcup U_{i}$ with $U_{i}$ contained in a $C^{1}$-embedded compact disk $U_{i}^{\#}$, and

$$
\left|D_{j} f(x)-D_{j} f(y)\right| \leq \delta_{i}^{*}(|x-y|)|x-y|^{k-1+\beta}
$$

for all $y \in U_{i}, x \in U_{i}^{\#}$, all $j$. Since $D_{j} f(y)$ is zero for $y \in U_{i}$, this yields an estimate for the derivative of $f$ on $U_{i}^{\#}$. Hence we may integrate $D f$ along a path $\gamma$ in $U_{i}^{\#}$ from $x$ to $y$ to obtain

$$
|f(x)-f(y)| \leq n \delta_{i}^{*}(|x-y|)|x-y|^{k-1+\beta}|\gamma|
$$

for $x \in U_{i}^{\#}, y \in U_{i}$, where $|\gamma|$ denotes the length of $\gamma, \delta_{i}^{*}(t)=C^{k-1+\beta} \delta_{i}(C t)$, and $C$ is the constant given in the following

Proposition. For any $C^{1}$-embedded compact disk $B$, there is a constant $C>0$ such that for all $x, y \in B$, there is a $C^{1}$ path in $B$ from $x$ to $y$ with length less than $C|x-y|$.

This is a consequence of the following inequalities: Let $\varphi: D \rightarrow \mathbf{R}^{n}$ be the embedding associated to $B$, where $D$ is the closed unit ball in $\mathbf{R}^{p}, p=\operatorname{dim} B$. Then there exist $r, s>0$ with

$$
r|a-b| \leq|\varphi(a)-\varphi(b)| \leq s|a-b| \text { for all } a, b \in D .
$$

The second inequality is immediate since $D \varphi$ is bounded on $D$; the first inequality follows by a straightforward argument, using the fact that $\varphi$ is a one-to-one immersion on a compact set. Now it is easy to establish the proposition, taking $C$ to be $s / r$.

In view of $(2)$ and this proposition, we may take $\varepsilon_{i}=n C \delta_{i}^{*}$ to complete the argument.

On $V$ : Some $g \in C^{k+\beta+}$ vanishing on $A$ is not critical at $x \in V$. By the $C^{k+\beta+}$ Inverse Function Theorem (see Appendix), $g^{-1}(0)$ is locally a $C^{k+\beta+}(n-$ 1)-submanifold containing $V$ in a neighborhood of $x$. $f$ restricted to this local submanifold satisfies the hypotheses of $\langle n-1, k\rangle$, and so we obtain the required Morse decomposition locally at $x \in V$. This gives the result, since the union of countably many Morse decompositions is again a Morse decomposition.

Proof of the Generalized Morse Theorem (i). For each $j=1, \ldots, m, D_{j} f$ is of class $C^{k-1+\beta+}$ and vanishes on $A$ since $f$ is critical on $A$. Applying the Vanishing Lemma we obtain

$$
\left|D_{j} f(x)\right| \leq \varepsilon_{j}^{\prime}(|x-y|)|x-y|^{k-1+\beta} \quad \text { for all } y \in A_{i}, x \in B_{i} \text {, all } j .
$$

As before, integrating along a path from $x$ to $y$ in $B_{i}$ yields

$$
|f(x)-f(y)| \leq \varepsilon_{i}(|x-y|)|x-y|^{k+\beta} \quad \text { for all } y \in A_{i} \text { and } x \in B_{i},
$$

where $\varepsilon_{i}$ is a suitable multiple of $\varepsilon_{i}^{\prime}$. Hence this is true in particular for all $x, y \in A_{i}$.

5. Proof of Theorem 1. For the proof we need a lemma.

LEMMA 1. (i) If $f \in C^{k+\beta+}\left(\mathbf{R}^{m}, \mathbf{R}^{n}\right), D f$ has rank 0 on $A$, and $A$ is $(k+\beta) n$ sigmafinite, then $f(A)$ is $n$-null.

(ii) If $f \in C^{k+\beta}\left(\mathbf{R}^{m}, \mathbf{R}^{n}\right)$, Df has rank 0 on $A$, and $A$ is $(k+\beta) n$-null, then $f(A)$ is n-null. 
(iii) If $f \in C^{k+\operatorname{Lip}}\left(\mathbf{R}^{m}, \mathbf{R}^{n}\right)$, Df has rank 0 on $A$, and $A$ is $(k+1) n$-null, then $f(A)$ is $n$-null.

PROOF (i). Since the countable union of null sets is null, we may assume that $A$ is $(k+\beta) n$-finite. Let $f_{i}$ be the $i$ th component of $f$. since $f_{1}, f_{2}, \ldots, f_{n}$ are all critical on $A$, the conclusion of the Generalized Morse Theorem applies for each $f_{i}$, hence for $f$, with respect to a Morse decomposition $A=\bigcup A_{i} . f\left(A_{0}\right)$ is countable, hence null. We show that $f\left(A_{j}\right)$ is null for each $j$, from which the conclusion follows since $f(A)=\bigcup f\left(A_{i}\right)$.

Fix $j$. Let $A_{j}=B$ for convenience of notation. By Theorem 3(i),

$$
|f(x)-f(y)| \leq \varepsilon(|x-y|)|x-y|^{k+\beta} \text { for all } x, y \in B .
$$

We wish to show that $\nvdash_{\alpha}^{n}(f(B)) \rightarrow 0$ as $\alpha \rightarrow 0$.

Choose $\theta>0$ so that $\varepsilon(\theta) \leq 1$. Then let $\eta=\min \left(\theta, \alpha^{1 /(k+\beta)}\right)$. Note that $\eta$ has the property that if $E \subset B$ and $\operatorname{diam}(E)<\eta$, then $\operatorname{diam} f(E)<\varepsilon(\eta) \eta^{k+\beta} \leq \alpha$, using (3).

Now

$$
\not_{\alpha}^{n}(f(B))=\inf \Sigma\left[\operatorname{diam}\left(C_{i}\right)\right]^{n}
$$

where the infimum is taken over all countable decompositions $\left\{C_{i}\right\}$ of $f(B)$ with $\operatorname{diam}\left(C_{i}\right)<\alpha$,

$$
\leq \inf \Sigma\left[\operatorname{diam} f\left(B_{i}\right)\right]^{n}
$$

where the infimum is taken over all countable decompositions $\left\{B_{i}\right\}$ of $B$ with $\operatorname{diam}\left(B_{i}\right)<\eta$. (The inequality holds because every decomposition $\left\{f\left(B_{i}\right)\right\}$ of $f(B)$ with $\operatorname{diam}\left(B_{i}\right)<\eta$ is a decomposition of the form $\left\{C_{i}\right\}$ above.)

$$
\begin{aligned}
& \leq \inf \Sigma\left[\varepsilon\left(\operatorname{diam} B_{i}\right)\left(\operatorname{diam} B_{i}\right)^{k+\beta}\right]^{n} \text { from (3) } \\
& \leq[\varepsilon(\eta)]^{n} \nvdash_{\eta}^{(k+\beta) n}(B) \text { by monotonicity of } \varepsilon \\
& \leq[\varepsilon(\eta)]^{n} \nvdash^{(k+\beta) n}(B) \rightarrow 0
\end{aligned}
$$

as $\eta$ (hence $\alpha$ ) tends to zero, since $B$ is $(k+\beta) n$-finite.

(ii) Let $A=\bigcup A_{i}$ be a Morse decomposition for $A$. Fix $j$ and let $B=A_{j}$. We show that $f(B)$ is $n$-null. Since every component of $f$ satisfies Theorem 3(ii), so does $f$; i.e.,

$$
|f(x)-f(y)| \leq M|x-y|^{k+\beta} \quad \text { for all } x, y \in B, \text { some } M>0 .
$$

Given $\alpha>0$, let $\eta=(\alpha / M)^{1 /(k+\beta)}$. Now

$$
\varkappa_{\alpha}^{n}(f(B))=\inf \Sigma\left[\operatorname{diam}\left(C_{i}\right)\right]^{n}
$$

where the infimum is taken over all countable decompositions $\left\{C_{i}\right\}$ of $f(B)$ with $\operatorname{diam}\left(C_{i}\right)<\alpha$,

$$
\leq \inf \Sigma\left[\operatorname{diam} f\left(B_{i}\right)\right]^{n}
$$

where the infimum is taken over all countable decompositions $\left\{B_{i}\right\}$ of $B$ with $\operatorname{diam}\left(B_{i}\right)<\eta$

$$
\begin{aligned}
& \leq \inf \Sigma\left[M\left(\operatorname{diam} B_{i}\right)^{k+\beta}\right]^{n} \text { by }(4) \\
& \leq M^{n} \nvdash_{\eta}^{(k+\beta) n}(B) \rightarrow 0 \text { as } \eta \rightarrow 0 \text { (hence as } \alpha \rightarrow 0 \text { ) }
\end{aligned}
$$

since $B$ is $(k+\beta) n$-null. 
(iii) Repeat the proof of (ii) with $\beta=1$, using Theorem 3(iii).

PROOF OF THEOREM 1(i). We can assume that $A$ is $((k+\beta)(n-r)+r)$-finite. Let $R_{i}=\left\{x \in A: \operatorname{rank} D f_{x}=i\right\}, i=0,1, \ldots, r$. We show that each set $f\left(R_{i}\right)$ is $n$-null. $f\left(R_{0}\right)$ is null by Lemma $1(\mathrm{i})$ since $(k+\beta)(n-r)+r \leq(k+\beta) n$.

Consider $p \in R_{j}$. It will suffice to find a neighborhood $U$ of $p$ such that $f\left(R_{j} \cap U\right)$ is $n$-null. By the $C^{k+\beta+}$ Inverse Function Theorem, there are coordinates in some neighborhood $U$ of $p$ so that $f\left(x_{1}, \ldots, x_{m}\right)=\left(x_{1}, \ldots, x_{j}, g\left(x_{1}, \ldots, x_{m}\right)\right)$, where $g \in C^{k+\beta+}\left(\mathbf{R}^{m}, \mathbf{R}^{n-j}\right)$. In these coordinates

$$
D f y=\left(\begin{array}{cc}
\operatorname{Id}_{j} & 0 \\
* & \left.D(g\rceil x_{1}, \ldots, x_{j}\right)
\end{array}\right)
$$

where $y=\left(x_{1}, \ldots, x_{m}\right)$ and $\left.g\right\rceil x_{1}, \ldots, x_{j}: \mathbf{R}^{m-j} \rightarrow \mathbf{R}^{n-j}$ is defined by

$$
g\rceil x_{1}, \ldots, x_{j}\left(x_{j+1}, \ldots, x_{m}\right)=g\left(x_{1}, \ldots, x_{m}\right) .
$$

Note that $\left.D(g\rceil x_{1}, \ldots, x_{j}\right)$ has rank zero for all $y \in R_{j}$.

For $C \subset \mathbf{R}^{m}$ and $\left(x_{1}, \ldots, x_{j}\right) \in \mathbf{R}^{j}$, denote by $C\left[x_{1}, \ldots, x_{j}\right]$ the set of points $\left(x_{j+1}, \ldots, x_{m}\right)$ in $\mathbf{R}^{m-j}$ such that $\left(x_{1}, \ldots, x_{m}\right) \in C$. (This is the "cross section of $C$ at $\left(x_{1}, \ldots, x_{j}\right)$ ".) Then $\left.g\right\rceil x_{1}, \ldots, x_{j}$ maps the rank 0 set $\left(U \cap R_{j}\right)\left[x_{1}, \ldots, x_{j}\right]$ onto $\left(f\left(U \cap R_{j}\right)\right)\left[x_{1}, \ldots, x_{j}\right]$.

Claim. For amost every $\left(x_{1}, \ldots, x_{j}\right)$, the cross section $\left(U \cup R_{j}\right)\left[\left(x_{1}, \ldots, x_{j}\right)\right]$ is $((k+\beta)(n-r)+r-j)$-finite, and hence $(k+\beta)(n-j)$-finite.

Assuming the claim, then by Lemma 1(i) applied to $g\rceil x_{i}, \ldots, x_{j}$, almost every cross section of $f\left(U \cup R_{j}\right)$ is $(n-j)$-null in $\mathbf{R}^{n-j}$. Hence by Fubini's theorem applied to $\mathbf{R}^{j} \times \mathbf{R}^{n-j}, f\left(U \cap R_{j}\right)$ is $n$-null.

To prove the claim, we refer to Federer [F1] (see [S3]), who has shown that for an arbitrary set $B$ in $\mathbf{R}^{m}$, if $B$ is $s$-finite then almost all cross sections $B\left[x_{1}, \ldots, x_{j}\right]$ are $(s-j)$-finite.

PROOF OF THEOREM 1(ii). Repeat the proof of Theorem 1(i) with " $\beta$ " replacing " $\beta+$ ", "null" replacing "finite", and "Lemma 1(ii)" replacing "Lemma $1(\mathrm{i}) "$.

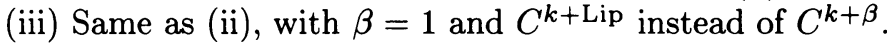

6. Examples. The hypotheses of Theorems 1 and 2 cannot be weakened. In the simplest case in which $k=1, n=1$, and $m=2$, Whitney's original example $f$ is, remarkably, already best possible: for $\beta=\log _{3} 4-1, f$ is $C^{1+\beta}$ and has a critical set $A$ which is $(1+\beta)$-finite, but $f(A)$ is not null. (By Theorem $2, f$ could not be $C^{1+\beta+}$, nor could $A$ be $(1+\beta)$-null.)

To see this, note that the curve $A$ (see $[\mathbf{W}]$ ) contains a self-similar Cantor set $C$. Scaling by a factor of three yields four copies of $C$, so its self-similarity dimension is $\log _{3} 4$ (see, e.g., [Ma or $\left.\mathbf{F a}\right]$ for a discussion of self-similarity dimension). This coincides as usual [Fa] with $\mathrm{HD}(C)$. In fact, all of $A$ is $\left(\log _{3} 4\right)$-finite, as the reader can show by considering a sequence of disk covers of $A$ with diameters tending to zero and bounded $\left(\log _{3} 4\right)$-measure.

From the construction of $f$ it is easy to show that there is a constant $K>0$ such that $|f(x)-f(y)| \leq K|x-y|^{\log _{3} 4}$ for all $x, y \in A$. From this and a $C^{k+\beta}$ version of the Whitney Extension Theorem (see $[\mathbf{N}]$ ) it follows that $f$ is of class $C^{\log _{3} 4}$.

We can obtain such an example for any other $\beta \in[0,1)$ by modifying the ratio of the size of any square to that of the next smaller square. Similarly, Whitney's 
higher-dimensional examples, mentioned in [W], will provide sharp counterexamples for $k=m-1>1$ as well.

Further examples can be constructed using the $(n-r)$-fold Cartesian product of $f$ with itself to show the following: for every $\theta>(k+\beta)(n-r)+r$ and $m \geq$ $(k+1)(n-r)+r$ there is a function $g \in C^{k+\beta}\left(\mathbf{R}^{m}, \mathbf{R}^{n}\right)$ and a set $B \subset \mathbf{R}^{m}$ of rank $r$ such that $B$ is $\theta$-null and $g(B)$ is not $n$-null. The details are in [N].

Finally, we mention the following result from $[\mathbf{N}]$, which shows that Whitneytype examples are in fact in great abundance. Call a topological arc $\gamma$ a quasi-arc if there is a $K>0$ such that for all $x, y \in \gamma$, the curve between $x$ and $y$ is contained in a ball of radius $K|x-y|$. Then any quasi-arc $\gamma$ with $\operatorname{HD}(\gamma)=s>1$, if $s$ is not an integer, is critical for some function $f \in C^{s}\left(\mathbf{R}^{n}, \mathbf{R}\right)$ not constant on $\gamma$. In particular, the same is true for any self-similar arc.

7. Comment. An even more delicate version of Theorem 2 relates the modulus of continuity $h(x)$ of $D^{k} f$ to a Hausdorff measure defined by using the gauge function $x^{k+n-1} h(x)$ rather than $x^{k+\beta+n-1}$ (see Rogers $[\mathbf{R}]$ for a discussion of these more general measures).

THEOREM. If

(i) $f \in C^{k}\left(\mathbf{R}^{m}, \mathbf{R}^{n}\right)$ and for each compact neighborhood $U$ in $\mathbf{R}^{m}$ there is an $M>0$ such that $\left|D^{k} f(x)-D^{k} f(y)\right|<M h(|x-y|)$ for all $x, y \in U$, where $h$ is monotone, continuous, and $h(0)=0$, and

(ii) $A$ is a critical set for $f$ which is $\left[x^{k+n-1} h(x)\right]-n u l l$, then $f(A)$ is null.

IDEA OF PROOF. Imitate the proof of Theorem 2 from the beginning, using appropriate versions of the Inverse Function Theorem and the Morse Vanishing Lemma.

Appendix. The $C^{k+\beta+}$ Inverse Function Theorem.

THEOREM. If $f \in C^{k+\beta+}\left(\mathbf{R}^{n}, \mathbf{R}^{n}\right), k \geq 1, \beta \in[0,1)$, and $D f_{x}$ is a linear isomorphism, then $f$ is invertible in a neighborhood of $x$ and $f^{-1}$ is of class $C^{k+\beta+}$.

Proof. The proof that $f^{-1}$ exists near $f(x)$ and is $C^{k}$ is the usual one (see, e.g., $[\mathbf{L}])$. The derivative satisfies

$$
D\left(f^{-1}\right)(y)=\left[D f\left(f^{-1}(y)\right)\right]^{-1} ; \quad \text { i.e., } \quad D\left(f^{-1}\right)=\operatorname{Inv} \circ D f \circ f^{-1},
$$

where Inv is the inverse map on $\operatorname{GL}(n)$. Since Inv is $C^{\infty}, D f$ is $C^{k-1+\beta+}$, and $f^{-1}$ is $C^{k}$, we may deduce that the composition is $C^{k-1+\beta+}$ from the

LEMMA. For $k \geq 1$, the composition of a $C^{k-1+\beta+}$ map $F$ with a $C^{k}$ map $G$ is $C^{k-1+\beta+}$.

Proof. By induction on $k$. The case $k=1$ is easy. For $k \geq 1, D F \circ G$ is $C^{k-2+\beta+}$ by the induction hypothesis, while $D G$ is $C^{k-1}$. Hence $D F \circ G \cdot D G=$ $D(F \circ G)$ is $C^{k-2+\beta+}$, and we are done.

\section{REFERENCES}

[Fa] K. J. Falconer, The geometry of fractal sets, Cambridge Tracts in Math. 85, Cambridge Univ. Press, 1985.

[F1] H. Federer, Some integralgeometric theorems, Trans. Amer. Math. Soc. 77 (1954), 242. 
[F2] _ Geometric measure theory, Grundlehren der Math. Wiss., vol. 153, Springer-Verlag, 1969.

[H] J. Harrison, Continued fractals and the Seifert conjecture, Bull. Amer. Math. Soc. (N.S.) 13 (1985), 147-153.

[HW] W. Hurewicz and H. Wallman, Dimension theory, Princeton Univ. Press, Princeton, N.J., 1941, p. 103.

[L] S. Lang, Analysis II, Addison-Wesley, Reading, Mass., 1969.

[Ma] B. Mandelbrot, The fractal geometry of nature, Freeman, San Francisco, Calif., 1982.

[M] A. P. Morse, The behavior of a function on its critical set, Ann. of Math. (2) 40 (1939), 62-70.

[N] A. Norton, Ph.D. Thesis, Univ. of California, Berkeley, 1986.

[R] C. A. Rogers, Hausdorff measures, Cambridge Univ. Press, 1970.

[S1] A. Sard, The measure of the critical values of differentiable maps, Bull. Amer. Math. Soc. 48 (1942), 883-890.

[S2] _ The equivalence of $n$-measure and Lebesgue measure on $E_{n}$, Bull. Amer. Math. Soc. 49 (1943), 758-759.

[S3] _ Images of critical sets, Ann. of Math. (2) 68 (1958), 247-259.

[S4] _ Hausdorff measure of critical images on Banach manifolds, Amer. J. Math. 87 (1965), 158-174.

[St] S. Sternberg, Lectures on differential geometry, Prentice-Hall, Englewood Cliffs, N.J., 1964.

[W] H. Whitney, A function not constant on a connected set of critical points, Duke Math. J. 1 (1935), 514-517.

[Y] Y. Yomdin, The geometry of critical and near-critical values of differentiable mappings, Math. Ann. 264 (1983), 495-515.

Department of Mathematics, University of CAlifornia, Berkeley, CaliforNIA 94720 